\title{
High-resolution multilocus sequence typing for Chlamydia trachomatis: improved results for clinical samples with low amounts of $C$. trachomatis DNA
}

Shlomo Pilo, Gal Zizelski Valenci, Mor Rubinstein, Lea Pichadze, Yael Scharf, Zeev Dveyrin, Efrat Rorman and Israel Nissan ${ }^{*}$ (iD

\begin{abstract}
Background: Several Multilocus Sequence Typing (MLST) schemes have been developed for Chlamydia trachomatis. Bom's MLST scheme for MLST is based on nested PCR amplification and sequencing of five hypervariable genes and ompA. In contrast to other Chlamydia MLST schemes, Bom's MLST scheme gives higher resolution and phylogenetic trees that are comparable to those from whole genome sequencing. However, poor results have been obtained with Bom's MLST scheme in clinical samples with low concentrations of Chlamydia DNA.

Results: In this work, we present an improved version of the scheme that is based on the same genes and MLST database as Bom's MLST scheme, but with newly designed primers for nested-1 and nested-2 steps under stringent conditions. Furthermore, we introduce a third primer set for the sequencing step, which considerably improves the performance of the assay. The improved primers were tested in-silico using a dataset of 141 Whole Genome Sequences (WGS) and in a comparative analysis of 32 clinical samples. Based on cycle threshold and melting curve analysis values obtained during Real-Time PCR of nested-1 \& 2 steps, we developed a simple scoring scheme and flow chart that allow identification of reaction inhibitors as well as to predict with high accuracy amplification success. The improved MLST version was used to obtain a genovars distribution in patients attending an STI clinic in Tel Aviv.

Conclusions: The newly developed MLST version showed great improvement of assay results for samples with very low concentrations of Chlamydia DNA. A similar concept could be applicable to other MLST schemes.
\end{abstract}

Keywords: Chlamydia trachomatis, Multilocus, Sequence, Typing, MLST, Melting curve analysis, Genovar

\section{Background}

Chlamydia trachomatis is a Gram-negative, obligate intracellular bacterium, responsible for a wide range of diseases [1]. Although C. trachomatis infections are often asymptomatic, late complications increase the risk of ectopic pregnancy and infertility when untreated $[2,3]$. In

\footnotetext{
* Correspondence: Israel.nissan@gmail.com
}

Ministry of Health, National Public Health Laboratory, Tel Aviv, Israel addition to being the most prevalent sexually transmitted bacteria worldwide, with an estimated annual 131 million new cases [4], C. trachomatis infections are associated with cervical cancer, and facilitate the transmission of HIV [5-8]. C. trachomatis DNA is comprised of a $\sim 1$ million base pairs (bp) long single circular chromosome, as well as multiple copies of a $7.5 \mathrm{~kb}$ long highly conserved plasmid [9-11]. Based on the antigenic properties of the

\section{$\triangle B M C$}

(c) The Author(s). 2021 Open Access This article is licensed under a Creative Commons Attribution 4.0 International License, which permits use, sharing, adaptation, distribution and reproduction in any medium or format, as long as you give appropriate credit to the original author(s) and the source, provide a link to the Creative Commons licence, and indicate if changes were made. The images or other third party material in this article are included in the article's Creative Commons licence, unless indicated otherwise in a credit line to the material. If material is not included in the article's Creative Commons licence and your intended use is not permitted by statutory regulation or exceeds the permitted use, you will need to obtain permission directly from the copyright holder. To view a copy of this licence, visit http://creativecommons.org/licenses/by/4.0/ The Creative Commons Public Domain Dedication waiver (http://creativecommons.org/publicdomain/zero/1.0/) applies to the data made available in this article, unless otherwise stated in a credit line to the data. 
major outer membrane protein (MOMP), C. trachomatis is typically divided into 17 distinct serovars [12-14], or 19 serovars which include subtypes [15]. The serovars include: trachoma serovars (A-C) that are the major etiological agents of preventable blindness; genital tract sexually transmitted serovars (D-K); and serovars L1-L3 that cause invasive urogenital infection or anorectal infection (lymphogranuloma venereum, LGV). Characterized by ulcerative proctitis [16], the LGV disease is of high concern $[12-15,17-19]$.

Since the advent of sequencing, sequence analysis of the MOMP gene (ompA), which encoded by nearly $1200 \mathrm{bp}$, has been widely used for epidemiological studies [20]. Genotypic variation within the ompA gene exhibits a great degree of polymorphism that cannot be identified by serotyping [21-23]. However, ompA variability does not provide sufficient discriminatory power for epidemiological purposes [12, 24]. Although C. trachomatis genome harbor regions with highly nucleotide diversity and high events of recombination, it is considerably conserved $[25,26]$. The genome is characterized by a low level of genetic diversity among variants $(<2 \%$ of the genome), and the ompA genotype classification strongly correlates with tissue tropism and disease outcome [26, 27].

Adding to the challenges described above, the C. trachomatis pathogen has an obligate intracellular life cycle. Clinical DNA samples contain only a small quantity of C. trachomatis residues mixed with DNA of both human cells and numerous diverse microorganisms. Hence, in order to obtain sufficient amounts of genomic DNA, for molecular typing, inoculation of clinical specimens and subsequently generating sufficient amounts of genomic DNA, requires an extensive in vitro culturing process, which may lead to genomic changes due, for example, to the absence of host immune pressures [12, 28-31].

In order to overcome the low discrimination power of the above-mentioned techniques, several genotyping systems have been developed [30]. Two groups developed multilocus sequence typing, based on the sequences of seven housekeeping genes (MLST-7) of C. trachomatis; Pannekoek el 2008 [32] and Dean et al. 2009 [33]. both schemes are supported by the PubMLST [34]. The MLST-7 method has been useful for exploring longterm and global epidemiological trends [13, 32, 35]. In order to explore partner tracing and molecular epidemiology of short-term outbreaks, a second multilocus sequence typing was developed, MLST-5 [36]. It is based on the investigation of five target variable regions of the Chlamydial genome: hctB, CT058, CT144, CT172 and $p b p B$. Although this method achieved high resolution it is not optimal for direct amplification from clinical samples. Therefore, the scheme was later improved by Bom et al. [28]. Bom's study used regions up to nearly $700 \mathrm{bp}$ in length for five target regions (the sixth target region is ompA $800 \mathrm{bp}$ ), improving PCR sensitivity, reducing PCR targets, and making it easier to assemble [28]. In a later study, additional advances were made in the method by redesigning two of the primers and adding M13-tailed primers [8]. The final protocol for primers and cycling conditions is described on the pubMLST website (http://mlstdb.bmc.uu.se/current.html), [34].

Despite these improvements clinical samples containing small quantities of Chlamydia DNA, as determined by GeneXpert (Xpert ${ }^{\circ} \mathrm{CT} / \mathrm{NG}$ ), did not show sufficient performance. Therefore, the aim of this study was to design novel primers for nested- 1 and nested-2 PCR, as well as a third primer set for the sequencing step. In addition, we developed a simple scoring scheme, based on the Real-Time PCR assay cycle threshold (CT) value and melting curve analysis, which predicted amplification success with high accuracy. Our method improved MLST results for clinical samples with very low amounts of $C$. trachomatis DNA. The methodology used during this study can be applied to other MLST schemes.

\section{Results}

The strategy that we used in our MLST primer designing is drawn in Fig. 1. In general, we constructed a consensus sequences of hctB (CT046), CT058, CT144, CT172, $p b p B$ (CT682) and ompA by running Clustal W with BioEdit 7.2.5 on datasets representing major and diverse genovars. On each consensus sequence, we localized the internal primer of Bom's [28] in order to use them as a flag. Our novel primers were located according to this flag and the specificity of each primer against the NCBI non-redundant (nr) database and Human RefSEqGene Sequences (RefSeq_Gene) using NCBI BLAST were tested. Accepted criteria for primer were as followed: identification of various serovars of $C$. trachomatis, and absence of human sequences. The primer list appears in Table 1 and the consensus gene and primer location in the supplementary information Figures S1, S2, S3, S4, S5 and S6. Using stringent criteria during primer designing, we selected primers that gave significantly better scoring by Clone Manager 9.0 (Sci-Ed Software) (supplementary Tables S1, S2). As a result, the average deviation per primer from the design criteria (as calculated by dividing the total number of deviation from the design criteria by the numbers of primers), in our scheme is 0.28 versus 2.13 in Bom's MLST scheme [28]. Comparative data about primers of Bom's MLST scheme and primers presented in this work appears in the supplementary information Tables S1, S2, S3, S4, S5 and S6.

In-silico analysis was performed in order to ensure the ability of the primers to amplify genes from a wide range of natural isolates, represented by 141 high quality 


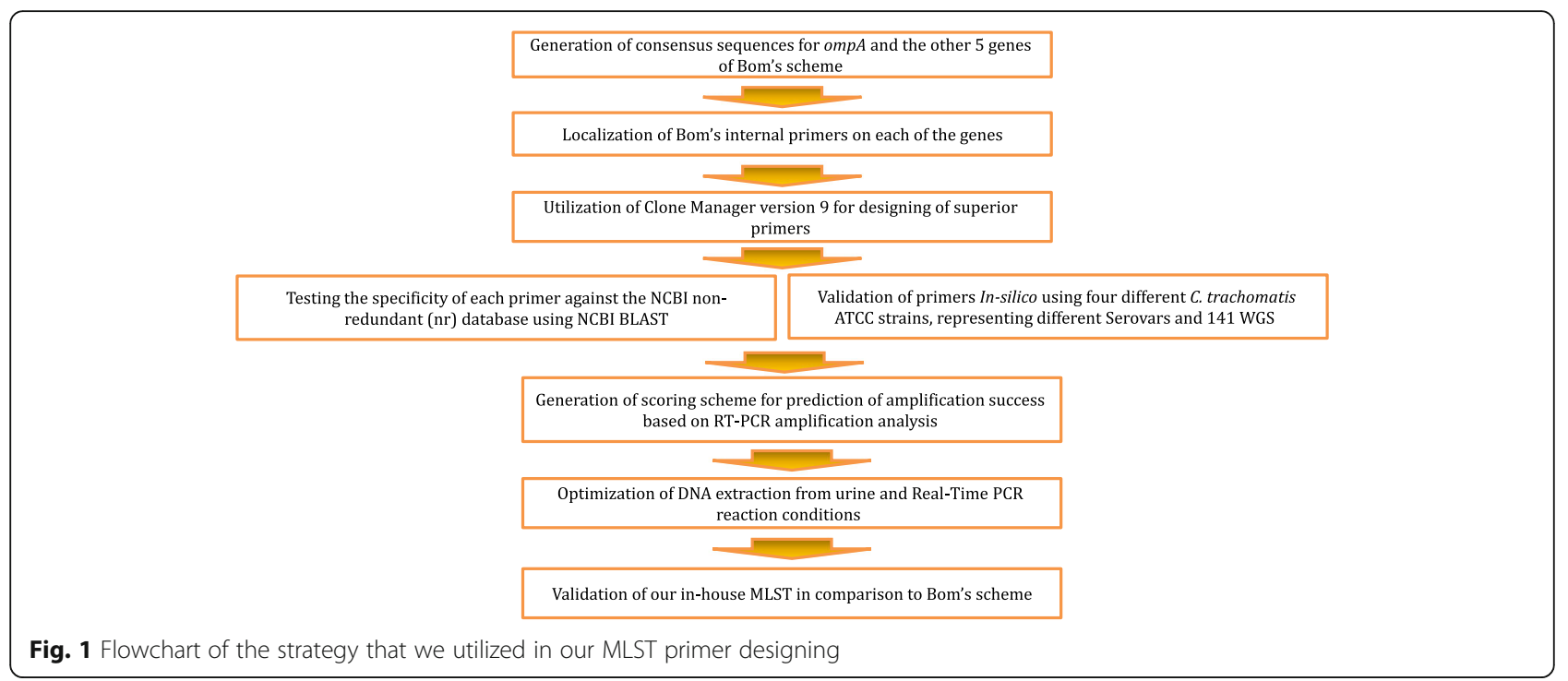

genome sequences of $C$. trachomatis from the NCBI Genome Sequences database. Using R script we calculated the binding and amplification potential of Bom's primers versus our newly designed primers. The analysis showed that the primer pairs were able to bind to the correct target, in a proper orientation and distance to allow efficient PCR amplification. The in-silico analysis confirmed that our primers were able to bind to a high proportion of the sequences with a very low number of mismatches (not more than two mismatches for a primer, supplementary information, Table S3, S4, S5 and S6).

To test our new primers for their ability to accomplish successful MLST, we selected four different ATCC strains of $C$. trachomatis representing different serovars ATCC VR-885 (D), ATCC VR-886 (J), ATCC VR-878 (G) and ATCC VR-901B (L1). Amplification and sequencing was carried out as described in the material and methods section. We noticed that regardless of the serovar, all these strains gave almost identical melting curves for the five genes of the MLST and ompA (Fig. 2A). We utilized this finding for the development of a scoring scheme for amplification success (Fig. 2B, next paragraph). To validate our MLST results, we downloaded the $C$. trachomatis ATCC strains sequences from the NCBI nucleotide gene bank (when available), and interrogated them for the alleles in the Uppsala MLST database (http://mlstdb.bmc.uu.se/current.html). The ompA genovar was confirmed by blastn search. The results were compared to STs and ompA genovars obtained by in-vitro amplification and sequencing using the same strains DNA. Identical results were obtained (Table 2). The ability of our method to achieve identical results to the Bom's MLST scheme [28] was further confirmed in a comparative study of 32 clinical samples (Table 3, supplementary information Table S8). Each sample was tested once. We used identical reaction condition between the two methods (Methods), except the specific primers, in order to be focused only in the primers contribution to the success rate of the method. The fit between the two methods was found to be excellent $(97.7 \%$ agreement in allele identification, $100 \%$ agreement in genovar identification). The newly designed method showed the best scores as shown in Table 3. The Limits of Detection (LOD) was determined to be 1-10 copies of chromosomal DNA using three $C$. trachomatis ATCC strains (Table 4).

We successfully developed scoring scheme for the prediction of amplification successes (material and methods and Fig. 2B). In $96.9 \%$ of 522 reactions, good agreement was found between the amplification qualities to the sequencing qualities (supplementary information Table S7). Notably, in only $3.1 \%$ of the samples no agreement was found, confirming that our scoring scheme can be used reliably. In general, this methodology can be applied to any MLST scheme. No significant correlation was found between the total nucleic acid concentrations in the sample to the sequencing success, representing the great challenge of obtaining Chlamydia sequences from a highly complex natural sample (Fig. 3a). Odds of success are high when $\mathrm{CT} \leq 33$ (89.5\%). Above this value, success rates decrease (Fig. 3b, c).

Based on the amplification score we developed a useful flow chart (supplementary information Figure S7), that allowed us to identify problems during the MLST (like the presence of reaction inhibitors, poor or no amplification, etc.) and to take corrective steps in order to improve the MLST success rate.

We applied our MLST scheme on 81 Chlamydia positive clinical urine samples as tested by GeneXpert collected during the years 2015-2016 from patients 
Table 1 MLST PCRs newly designed primer list. The colors signify the different groups of primers: light blue, external gene position (Nested-1). Yellow, internal gene position (Nested-2). White, primers used for sequencing

\begin{tabular}{|c|c|c|c|c|c|c|c|c|}
\hline Number & Gene & $\begin{array}{l}\text { Gene } \\
\text { Position }\end{array}$ & Primer name & Sequence $5^{\prime}-3^{\prime}$ & $\mid \begin{array}{c}5 . \\
\text { Chrom-position } \\
\text { (1) }\end{array}$ & $\begin{array}{l}\text { Primer } \\
\text { Length }\end{array}$ & $\mathrm{Tm}$ & $\begin{array}{l}\text { Search against NCBI } \\
\text { Blast Human } \\
\text { RefSeq_Gene } \\
\text { (S//\%/E), (2) }\end{array}$ \\
\hline 1 & \multirow{6}{*}{ CT172 } & External & CT172_CONF & CAGTGACTTGATTGGATAAC & 195282 & 20 & 55 & $\begin{array}{c}30.2,15 / 15 \mathrm{bp}, 100 \%, \\
E=2\end{array}$ \\
\hline 2 & & External & CT172_CONR & TGATCAAGCCATCTTAGAC & 195799 & 19 & 55 & $\begin{array}{c}32.2,16 / 16 \mathrm{bp}, 100 \%, \\
\mathrm{E}=0.39\end{array}$ \\
\hline 3 & & Internal & CT172_new-F & TAAAGGTCGCCCAAATTCC & 195373 & 19 & 59 & $\begin{array}{c}30.2,15 / 15 \mathrm{bp}, 100 \%, \\
\mathrm{E}=1.5\end{array}$ \\
\hline 4 & & Internal & CT172_new-R & GCTCCGGCTATTTTGTTTAGG & 195778 & 21 & 61 & $\begin{array}{c}32.2,16 / 16 \mathrm{bp}, 100 \% \text {, } \\
\mathrm{E}=0.64\end{array}$ \\
\hline 5 & & Seq_F & CT172-seqF & AAATTCCATGTTATAATC & 195385 & 18 & 45 & $\begin{array}{c}32.2,16 / 16 \mathrm{bp}, 100 \%, \\
\mathrm{E}=0.39\end{array}$ \\
\hline 6 & & Seq_R (3) & CT172-seqR & TATTTCCCTCAGTTATTC & 195756 & 18 & 49 & $\begin{array}{c}36.2,18 / 18 \mathrm{bp}, 100 \%, \\
E=0.025\end{array}$ \\
\hline 7 & \multirow{6}{*}{ pbpB (CT682) } & External & CT682_CONF & TGGGATTTATTATTGCTTTG & 780726 & 20 & 53 & $\begin{array}{c}34.2,17 / 17 \mathrm{bp}, 100 \%, \\
\mathrm{E}=0.13\end{array}$ \\
\hline 8 & & External & CT682_CONR & TACACCTAATTTGCCGATCA & 781453 & 20 & 58 & $\begin{array}{c}30.2,15 / 15 \text { bp, } 100 \%, \\
E=2\end{array}$ \\
\hline 9 & & Internal & CT682_new_F & TCATCACTTTGCGTATATGG & 780750 & 20 & 57 & $\begin{array}{c}30.2,15 / 15 \text { bp, } 100 \%, \\
E=2\end{array}$ \\
\hline 10 & & Internal & CT682_new_R & ATAGGCATGCATCTCTAAC & 781420 & 19 & 56 & $\begin{array}{c}32.2,16 / 16 \mathrm{bp}, 100 \%, \\
\mathrm{E}=0.39\end{array}$ \\
\hline 11 & & Seq_F & CT682-seqF & TGGCATGTTGCTGTTGTG & 780767 & 18 & 60 & $\begin{array}{c}34.2,17 / 17 \mathrm{bp}, 100 \%, \\
E=0.097\end{array}$ \\
\hline 12 & & Seq_R & CT682-seqR & СGACGCTCGATATTCCTG & 781376 & 18 & 59 & $\begin{array}{c}28.2,14 / 14 \mathrm{bp}, 100 \%, \\
E=6\end{array}$ \\
\hline 13 & \multirow{6}{*}{ ompA } & External & ompA_CONF & AAGATAGCGAGCACAAAGAG & 780107 & 20 & 59 & $\begin{array}{c}30.2,15 / 15 \mathrm{bp}, 100 \%, \\
\mathrm{E}=2\end{array}$ \\
\hline 14 & & External & ompA_CONR & ACTGCGTATTTGTCTGCATC & 778943 & 20 & 60 & $\begin{array}{c}30.2,15 / 15 \mathrm{bp}, 100 \%, \\
E=2\end{array}$ \\
\hline 15 & & Internal & ompA_new-F & CTCTTGAAATCGGTATTAG & 780051 & 19 & 51 & $\begin{array}{c}30.2,15 / 15 \text { bp, } 100 \%, \\
E=1.5\end{array}$ \\
\hline 16 & & Internal & ompA_new_R2 & CTTGCTCGAGACCATTTAAC & 779174 & 20 & 58 & $\begin{array}{c}30.2,15 / 15 \text { bp }, 100 \%, \\
E=2\end{array}$ \\
\hline 17 & & Seq_F & ompA-seq_F2 & TTGCCGCTTTGAGTTCTG & 780029 & 18 & 60 & $\begin{array}{c}30.2,15 / 15 \text { bp, } 100 \%, \\
E=1.5\end{array}$ \\
\hline 18 & & Seq_R & ompA-seq_R2 & GAGAGCTAAACTTGCTTG & 779230 & 18 & 55 & \begin{tabular}{|c|}
$30.2,15 / 15 \mathrm{bp}, 100 \%$ \\
$E=1.5$
\end{tabular} \\
\hline 19 & \multirow{6}{*}{ hctB (CT046) } & External & CT046_CONF & ACAACGTTTCCTCGAAGAC & 51209 & 19 & 59 & $\begin{array}{c}28.2,17 / 18 \text { bp, } 94 \%, \\
E=6\end{array}$ \\
\hline 20 & & External & CT046_CONR & GAAGCGCAAACCTTTACAC & 52030 & 19 & 59 & \begin{tabular}{|c}
$28.2,14 / 14$ bp, $100 \%$, \\
$E=6$
\end{tabular} \\
\hline 21 & & Internal & CT046_new-F2 & ATGGGCACATTTGGACATTG & 51239 & 20 & 61 & $\begin{array}{c}32.2,16 / 16 \mathrm{bp}, 100 \%, \\
\mathrm{E}=0.51\end{array}$ \\
\hline 22 & & Internal & CT046_new-R2 & TACACCAGAAGCAGCTACAC & 52016 & 20 & 61 & $\begin{array}{c}\begin{array}{c}32.2,16 / 16 \mathrm{bp}, 100 \%, \\
\mathrm{E}=0.51\end{array} \\
\end{array}$ \\
\hline 23 & & Seq_F & CT046_seq_F2 & AAGAAGAGTTGCCTTACC & 51280 & 18 & 55 & $\begin{array}{c}32.2,16 / 16 \text { bp, } 100 \%, \\
E=1.5\end{array}$ \\
\hline 24 & & Seq_R & CT046_seq_R2 & CAAGCTGCAGTATGTTTATG & 51991 & 20 & 56 & $\begin{array}{c}\begin{array}{c}32.2,16 / 16 \text { bp }, 100 \%, \\
E=0.51\end{array} \\
\end{array}$ \\
\hline 25 & \multirow{6}{*}{ CT144 } & External & CT144_CONF & AAGGAGGCAGATTAATGAC & 160680 & 19 & 56 & $\begin{array}{c}\begin{array}{c}34.2,17 / 17 \mathrm{bp}, 100 \%, \\
E=0.097\end{array} \\
\end{array}$ \\
\hline 26 & & External & CT144_CONR & TCCTGAAGAAGTTGCATAG & 161467 & 19 & 56 & $\begin{array}{c}32.2,16 / 16 \mathrm{bp}, 100 \%, \\
E=0.39\end{array}$ \\
\hline 27 & & Internal & CT144_new_F & AACAGCAACAGCCAATTAC & 160810 & 19 & 58 & $\begin{array}{c}32.2,16 / 16 \mathrm{bp}, 100 \%, \\
\mathrm{E}=0.39\end{array}$ \\
\hline 28 & & Internal & CT144_new_R & TATACCATGTGGACCCTAAAC & 161414 & 21 & 58 & $\begin{array}{c}32.2,16 / 16 \mathrm{bp}, 100 \%, \\
\mathrm{E}=0.64\end{array}$ \\
\hline 29 & & Seq_F & CT144_seqF & TCGTGACTAATCCGAAATC & 160908 & 19 & 56 & $\begin{array}{c}28.2,14 / 14 \text { bp, } 100 \%, \\
E=6\end{array}$ \\
\hline 30 & & Seq_R & CT144_seqR & CTAAACATACGGCTATTCC & 161399 & 19 & 54 & $\begin{array}{c}34.2,17 / 17 \mathrm{bp}, 100 \%, \\
E=0.097\end{array}$ \\
\hline 31 & \multirow{6}{*}{ Ст058 } & External & CT058_CONF & TAAGCACAGCAGGGAATG & 67871 & 18 & 58 & $\begin{array}{c}32.2,16 / 16 \mathrm{bp}, 100 \% \text {, } \\
\mathrm{E}=0.32\end{array}$ \\
\hline 32 & & External & CT058_CONR & TGCGTGATTGAGGTCTTG & 68754 & 18 & 59 & $\underset{E=6}{28.2,14 / 14 b p, 100 \%,}$ \\
\hline 33 & & Internal & CT058_new_F2 & ААТССTCСTTGGCCTCTC & 67903 & 18 & 60 & \begin{tabular}{|c|}
$32.2,16 / 16 \mathrm{bp}, 100 \%$, \\
$\mathrm{E}=0.39$
\end{tabular} \\
\hline 34 & & Internal & CT058_new_R2 & TTGGAAGGCGCGGATAAC & 68739 & 18 & 62 & $\begin{array}{c}30.2,15 / 15 \mathrm{bp}, 100 \%, \\
E=1.5\end{array}$ \\
\hline 35 & & Seq_F & CT058_seqF & TTGGCCTGAAGTAGAGAC & 67995 & 18 & 57 & \begin{tabular}{|c}
$\begin{array}{c}32.2,16 / 16 \mathrm{bp}, 100 \%, \\
\mathrm{E}=0.39\end{array}$ \\
\end{tabular} \\
\hline 36 & & Seq_R & CT058_seqR & AAAGGTGGCTGCGTTAAG & 68637 & 18 & 59 & $\begin{array}{c}30.2,15 / 15 \mathrm{bp}, 100 \%, \\
E=1.5\end{array}$ \\
\hline
\end{tabular}

(1) In the genome of Chlamydia trachomatis D/UW-3/CX ACCESSION NC_000117.

(2) Score (S), Identities (I), Present Identities (\%), Expect (E).

(3) For this specific primer a single hit of complete identity was found in the Human genome. Nevertheless, this is a sequencing primer that is not part of the amplification steps and its in-vitro performance was found to be very good. 

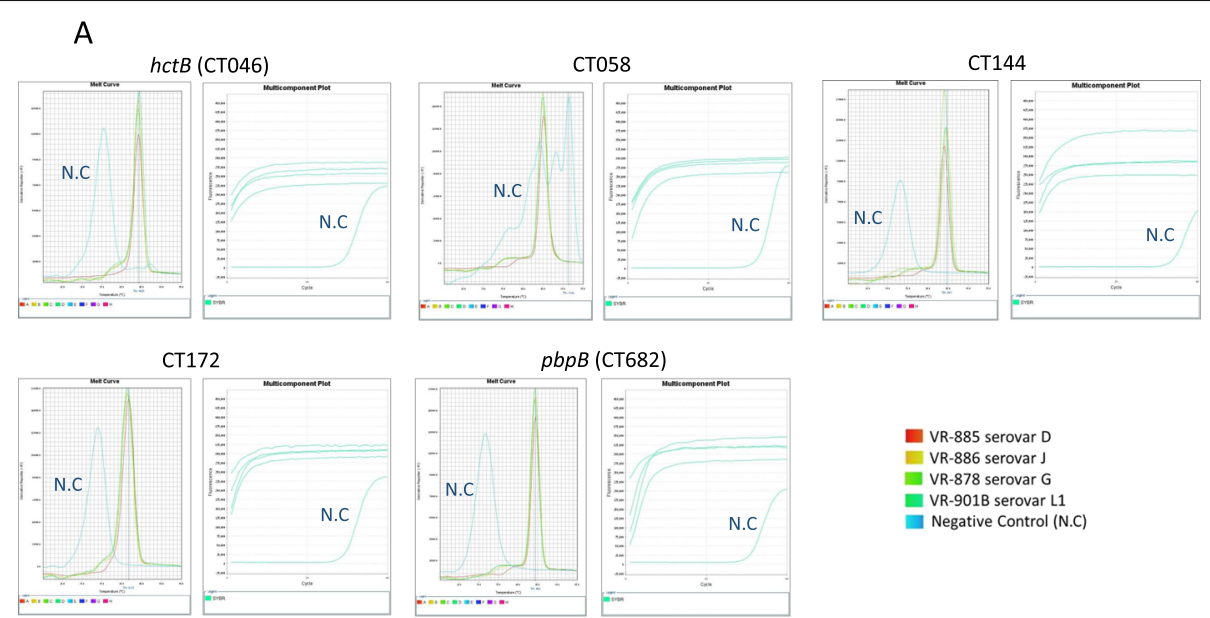

B
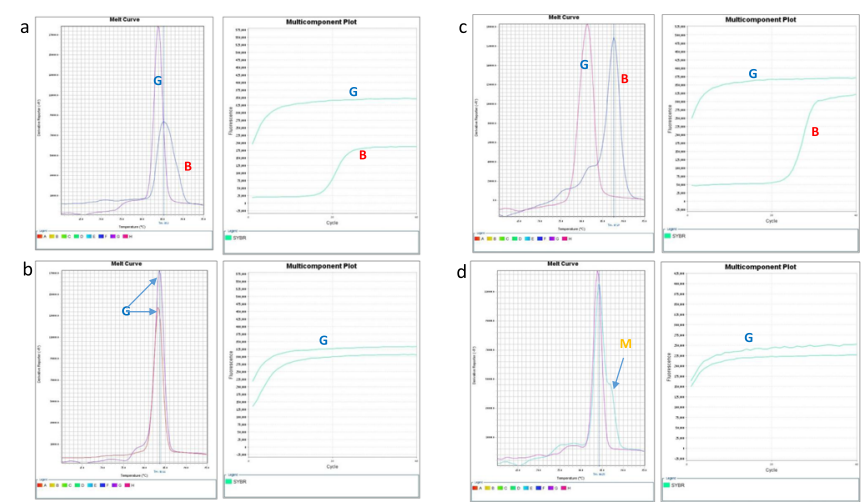

G- good M- moderate B-bed

Fig. 2 Representative results for typical nested 2 step amplification and melting curve analysis for each of the hctB (CT046), CT058, CT144, CT172 and $p b p B$ (CT682) genes, the left figure shows the melting curve and the right the amplification plot (multicomponent plot). A) typical successful nested 2 step PCR amplification of the ATCC strains: ATCC VR-885, ATCC VR-886, ATCC VR-878 and ATCC VR-901B. B) Typical successful and unsuccessful nested 2 step PCR amplification of clinical samples: G (Good) successful amplification, B (Bad) Unsuccessful amplification and M (Moderate). Unsuccessful amplification of CT058 of sample 298 versus positive control (a), Successful amplification of CT046 of sample 293 versus positive control (b), Unsuccessful amplification of CT172 of sample 230 versus positive control (c), and Moderate amplification of CT682 of sample 362 versus positive control $(d)$

attending a community clinic for STI's and HIV in TelAviv (supplementary information Table S9). The genovars distribution and the correlation between genovar and sequence types (ST) of the clinical samples appear in Fig. 4a and b respectively. The patients attending this clinic were not required to identify themselves. The provision of personal information, including sexual preference, sex, age, etc., had permission solicited but the details were not verified. We were able to obtain ompA sequences from 67 out of 81 urine samples (82\%) and complete MLST data (five genes) for 52 out of 81 samples (64.2\%). Interestingly 5 samples included alleles combination that did not appear in the Uppsala database therefore represents novel STs. For example, sample number 296 contains the following alleles combination: hctB-10, CT058-6, CT144-7, CT172-2, CT682-1. The appearance of hctB-10, CT058-6 in the combination with the other alleles is novel. The three most prevalent genotypes were $\mathrm{E}(n=34 ; 51 \%), \mathrm{G}(n=9 ; 13 \%)$, and $\mathrm{D}(n=$ $8 ; 12 \%)$. The STs of the samples were highly diverse with ST52, 56 \& 109 appearing at higher numbers (Fig.4b). LGV genotypes were not detected in this samples set.

The amount of DNA template in the nested-1 step was very important and we found that we can obtain good sequences in several cases of failure, by increasing the volume of nested-1 to $40 \mu \mathrm{l}$ thus doubling the volume of template DNA (from $8 \mu \mathrm{l}$ to $16 \mu \mathrm{l}$ ) (not presented).

\section{Discussion}

The aim of this study was to design a new nested PCR MLST scheme based on Bom's MLST scheme [28], 
Table 2 Sequencing of C. trachomatis ATCC strains. Sequences were downloaded from the NCBI nucleotide gene bank (when available) or determined de novo using our in-house MLST scheme. The alleles were determine by Uppsala MLST database interrogation and the ompA genovar was determined by Blast search against the nr NCBI database. In all cases, the results of the de novo sequencing were identical to the sequence downloaded from the NCBI database

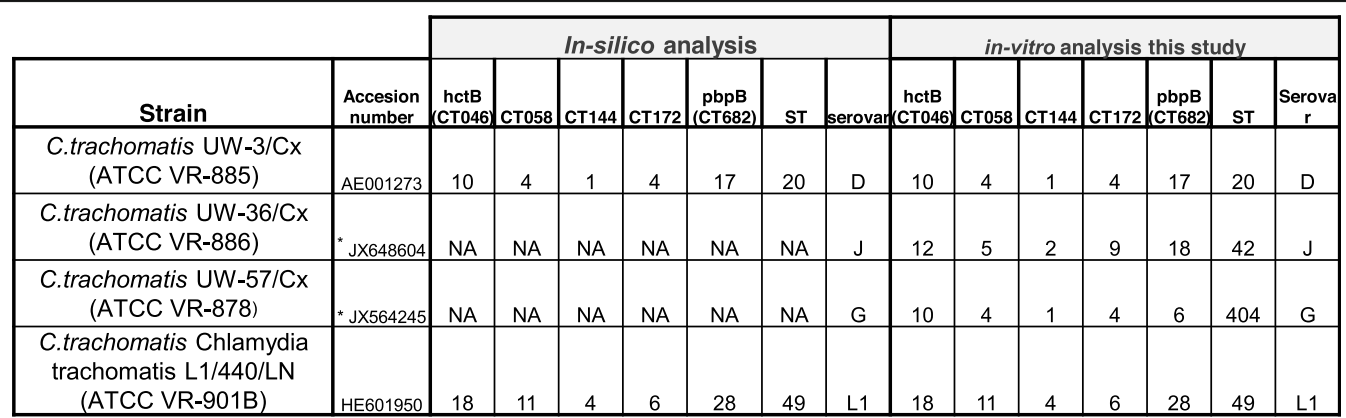

Numbers in the table for $h c t B$ (CT046), CT058, CT144, pbpB (CT682), represents allele designation

(*) High quality genome sequence is not available (July 132020 ). The sequence of ompA only was available

at the NCBI nucleotide gene bank.

NA-Sequence is not available

making it more suitable for clinical samples containing a large proportion of irrelevant DNA (from human and diverse microorganisms) with only a small amount of Chlamydia DNA. To achieve this goal, we redesigned nested-1 and nested-2 PCR primers using highly stringent criteria, and introduced a third primer set for the sequencing step. Using clinical urine samples, our version identified the same alleles as the original Bom's MLST scheme [28] in almost all of the cases (97.7\%), while allowing identification of a higher number of alleles (13.8\% increase), Table 3. Nevertheless, comparative analysis of more and divers samples (from different body sites, paper in preparation) are needed in order to support the superiority of our method.

This study showed that careful planning of nested-1 and nested-2 and sequencing primers and the utilization of various in-silico analyses, enabled improvement in amplification and sequencing success rate. In a typical thermocycler, the success or failure of reaction cannot be predicated and is apparent only after accepting the sequence. Therefore, the ability to apply changes or corrections is very limited. On the contrary, our method allow efficient real-time intervention as needed in order the retrieve the maximal information from the sample. By using this method during the procedure, the user can take a calculated decision (Figure S7). The development of a scoring scheme and flow chart to assess amplification success allowed us to overcome cases of reaction inhibitors, poor or no amplification and to improve the MLST performance. In fact, a similar scoring scheme can be applied to any MLST scheme. Note that no high resolution melting analysis (HRMA) was used nor melting curve results, for purposes of genotyping as described by Giffard PM et al., 2018 regarding CtGEM typing method [37].

Our MLST scheme was used successfully for analysis of various clinical samples including vaginal and rectal swabs (not shown). By adjusting the amount of nucleic acid taken for nested PCR-1, as described in the material and methods section, most of the PCR inhibition was prevented. This study presents for the first time data about Israel genovars and ST's distribution among $C$. trachomatis. Notably, the most prevalent genotype of isolates from patients attending an STI clinic in Tel Aviv is genotype E, which accounts for about half of the isolates. Interestingly, the genovar's distributions are similar to the report from European countries [24, 27] and Tunisia [38], but present differences from Asian countries such as China, where the genotypes D and G were the most common [39], and India where genotype D was found to be most prevalent [40].

In the paper of Björn Herrmann et al., (2015) he describe the genovars and STs distribution among 2089 specimens from 16 countries [24]. In that work they found that the most common STs among heterosexuals are 3, 12, 55 \& 56 and in men having sex with men (MSM) the predominating STs are 52, 58, 108 \& 109. Our data shows that the predominate STs in Israel are 52, 56 and 109 and this is the first report from Israel. Our data expand the knowledge about the global distribution of these strains.

The novel MLST scheme presented in this paper was adopted as the official working procedure of the Chlamydia Reference Laboratory in the Israeli National Public Health Laboratories, Tel-Aviv.

\section{Study limitations}

For the comparative analysis, we used 32 samples (from total of the 81 samples). This is a relatively small number. In the real world, the performance of the assay might be affected by sampling, transport condition and other unknown factors. Theoretically, in other sets of samples the success rate might be different. Analyzing 
Table 3 Comparative results of Bom's MLST scheme [28], and this study on 32 positive clinical samples that were obtained from a community clinic for STI's and HIV in Tel-Aviv. The total number of alleles (except ompA) is 160 (=32X5)

\begin{tabular}{|c|c|c|c|c|c|c|c|}
\hline Method & $\begin{array}{c}\text { Number } \\
\text { of } \\
\text { samples }\end{array}$ & $\begin{array}{c}\text { Identification } \\
\text { of ST, (\%) }\end{array}$ & $\begin{array}{c}\text { 1Total alleles } \\
\text { identified with } \\
\text { exact match } \\
\text { (\%) }\end{array}$ & $\begin{array}{c}\text { Identification of } \\
\text { genovar (\%) }\end{array}$ & $\begin{array}{c}\text { Agreement in ST } \\
\text { (for complete } \\
\text { genotyping) }\end{array}$ & $\begin{array}{c}\text { 2Agreement in } \\
\text { alleles identification } \\
\text { (\%) }\end{array}$ & $\begin{array}{c}\text { Agreement in } \\
\text { genovars }\end{array}$ \\
\hline $\begin{array}{c}\text { Bom's } \\
\text { MLST } \\
\text { Scheme }\end{array}$ & 32 & $13(40.6)$ & $86(53.75)$ & $22(68.8)$ & 13 of $13(100 \%)$ & 85 of $87(97.7)$ & 20 of 20 (100\%) \\
\cline { 1 - 4 } This study & 32 & $17(53.1)$ & $108(67.5)$ & $23(71.9)$ & & \\
\hline
\end{tabular}

1. Exact match to allele in Uppsala database.

2. Agreement in alleles assignment (the highest score by Uppsala database), including those that are not in exact match.

more samples from diverse sources (not just urine) will allow us to test the robustness of this new method in comparison to other MLST schemes. Nevertheless, insilico test of our primers on a wide range of C. trachomatis whole genomes demonstrated the primers potential for sequencing a wide variety of $C$. trachomatis natural isolates.

Additional limitation was the use of different reaction volumes from the Bom's MLST scheme [28]. While we used reaction volumes of $20 \mu \mathrm{l}$ and $10 \mu \mathrm{l}$ for nested- 1 and neasted- 2 respectively, Bom's MLST method presents $25 \mu \mathrm{l}$ reaction volumes for both nested-1 and nested- 2 . As indicated in the material and method and in the results sections, we used identical reaction condition for both methods, except the primers, in order to focus the study in the primers contribution to the success rate of the process.

\section{Conclusions}

Taken together, in-silico and in-vitro comparison of our redesigned primers verses Bom et al. 2011 primers [28], found that our novel primers performed better. Notably, Bom et al. 2013 published a redesign of the pBpB 2366 $\mathrm{R}$ and CT $1678 \mathrm{R}$ primers in order to improve their specificity [8]. This emphasizes the importance of careful primers designing. In conclusion: Our method introduces many advantages to the Bom's MLST scheme. Many of the principles developed during this study can be easily adjusted to other MLST schemes, such as Chlamydia MLST-7 or any other bacterium's MLST determination.

\section{Methods}

\section{DNA and clinical samples}

DNA of the following strains were purchased from the ATCC (https://www.atcc.org/): ATCC VR-885 (D), ATCC VR-886 (J), ATCC VR-878 (G) and ATCC VR901BD (LGV1).

81 C. trachomatis positive urine samples, collected during the years $2015-16$ were stored at $-80{ }^{\circ} \mathrm{C}$ until DNA extraction.

All samples were examined by GeneXpert (Cepheid, Xpert $^{\circ} \mathrm{CT} / \mathrm{NG}$ ) with a reagent kit for detection and differentiation of Chlamydia trachomatis and Neisseria gonorrhoeae (catalog number GXCT/NG-CE-10), used according to the instruction of the manufacturer.

\section{Primer design}

The general scheme is described in Fig. 1. In details, for each target gene DNA sequences were downloaded from

Table 4 Limits of Detection (LOD) for each of the hctB (CT046), CT058, CT144, CT172, pbpB (CT682) and ompA genes, using the new primers schemes, of the ATCC strains: VR-885, VR-878 and VR-901B. + Positive detection. - Negative detection. The LOD is between 1 and 10 genomic copies (green)

\begin{tabular}{|c|c|c|c|c|c|c|c|c|c|c|c|c|c|c|c|c|c|c|c|}
\hline \multirow[b]{2}{*}{ Dilution } & \multirow{2}{*}{\begin{tabular}{|l|} 
Gene \\
*Copy \\
number \\
\end{tabular}} & \multicolumn{3}{|c|}{ Ст046 } & \multicolumn{3}{|c|}{ СТ058 } & \multicolumn{3}{|c|}{ CT144 } & \multicolumn{3}{|c|}{ CT172 } & \multicolumn{3}{|c|}{ CT682 } & \multicolumn{3}{|c|}{ ompA } \\
\hline & & 885 & 878 & 901 & 885 & 878 & 901 & 885 & 878 & 901 & 885 & 878 & 901 & 885 & 878 & 901 & 885 & 878 & 901 \\
\hline \begin{tabular}{|l|}
$10^{\wedge}-1$ \\
\end{tabular} & 11400 & + & + & + & + & + & + & + & + & + & + & + & + & + & + & + & + & + & + \\
\hline $10^{\wedge}-2$ & 1140 & + & + & + & + & + & + & + & + & + & + & + & + & + & + & + & + & + & + \\
\hline $10^{\wedge}-3$ & 114 & + & + & + & + & + & + & + & + & + & + & + & + & + & + & + & + & + & + \\
\hline $10^{\wedge}-4$ & 11.4 & + & + & + & + & + & + & + & + & + & + & + & + & + & + & + & + & + & + \\
\hline $10^{\wedge}-5$ & 1.14 & + & - & + & + & - & + & + & - & - & + & + & + & + & - & - & - & - & - \\
\hline $10^{\wedge}-6$ & 0.114 & - & - & - & - & - & - & - & - & - & - & - & - & - & - & - & - & - & - \\
\hline $10^{\wedge}-7$ & 0.0114 & - & - & - & - & - & - & - & - & - & - & - & - & + & - & - & - & - & - \\
\hline
\end{tabular}

*Calculated copy number in $8 \mu \mathrm{l}$ added to the reaction 

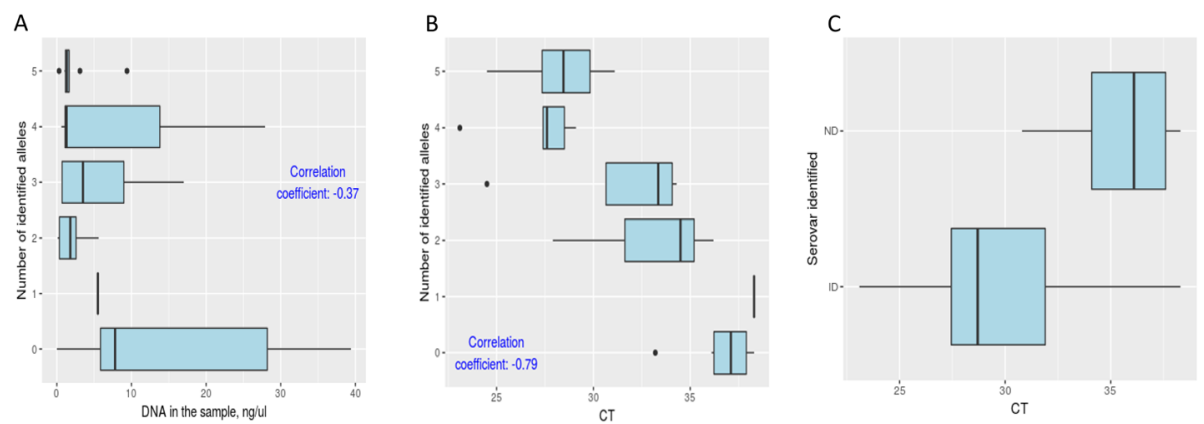

Fig. 3 The correlation between DNA concentration $(\mathbf{a})$ and threshold cycle $(\mathbf{b}, \mathbf{c})$ in the sample to sequencing successes are shown in the boxplots. c Correlation between threshold cycle to sequencing successes of ompA. Odds of success are high when CT $\leq 33$. Above this value, success rates decrease

NCBI, the DNA representing various genovars (urogenital, LGV). A consensus sequence was created using Clustal W (BioEdit ver 7.2.5). The consensus sequences were uploaded into Clone Manager 9.0 (Sci-Ed Software) and the internal primers of Bom's [28] scheme were located on the consensus in order to serve as a flag for localization of the new primers. We used stringent criteria (supplementary information, Tables S1, S2) to design new primers. Tables S1, S2 include detailed information on each primer. The consensus sequences for the MLST-5 and $о m p A$, including the position of the primers appear in the supplementary information Figure S1, S2, S3, S4, S5 and S6. The specificity of each primer was further tested by running blastn against the NCBI Human RefSeqGene Sequences (RefSeq_Gene) database and by in-silico amplification analysis against 141 C. trachomatis WGS (supplementary information, Tables S3, S4, S5 and S6).

\section{DNA extraction}

Nucleic acids were extracted from $1.5 \mathrm{ml}$ of Chlamydiapositive urine samples, using the MagNA pure compact
Instrument (Roche), according to the manufacturer's instructions. In short, frozen urine samples were left to thaw at room temperature. Thawed samples were centrifuged for $10 \mathrm{~min}$ at20,000 g. At the end of the centrifugation most of the supernatant was removed keeping $200 \mu \mathrm{l}$ for resuspension of the pellets. $200 \mu \mathrm{l}$ of MagNa Pure Lysis Buffer (catalog number 04659180001) and $20 \mu \mathrm{l}$ of Proteinase K (Roche, 04909640001) were added and the samples were incubated at $65^{\circ} \mathrm{C}$ and $95^{\circ} \mathrm{C}$ for $10 \mathrm{~min}$ each. After lysis the lysate was left to cool down at room temperature for $5 \mathrm{~min}$. $400 \mu \mathrm{l}$ of lysate were loaded into the MagNA Pure Compact Instrument (Roche) and the nucleic acids were extracted using MagNA Pure Compact Nucleic Acid Isolation Kit I (Roche, catalog number 03730964001). The nucleic acids were eluted in $100 \mu \mathrm{l}$.

\section{Measurement of the DNA concentration}

The concentrations of the nucleic acids extracted from clinical samples were measured using nanodrop 2000. Magnetic beads purified DNAs after the nested-2 step
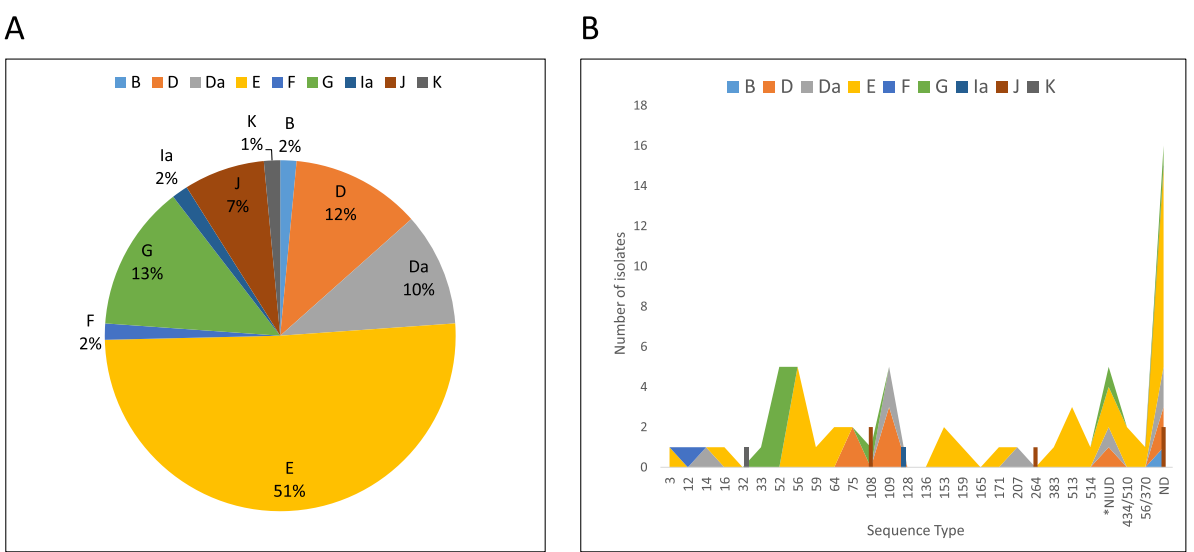

Fig. 4 Genovars and MLST distribution of the 81 clinical samples from patients attending community clinic for STI's and HIV in Tel-Aviv. a Serovar of 67 out of 81 urine samples (82\%). b Integration of the genovars and MLST distribution of 52 out of 81 samples (64.2\%). NIUD - Not In Uppsala Database, represent novel ST's. ND- Not Determined. STIST - there are two options for sequence type $(4341510,56 \backslash 370)$ 
PCR (as described at the "Cleaning PCR products" section) were measured using the DeNovix ds broad range kit (catalog KIT-DSDNA-BROASD-2) with DeNovix QFX Fluorometer or by the PicoGreen (Invitrogen, P11496) method using TECAN SPECTRAFOUR Plus, according to manufacturer's instructions. The nucleic acids DNA concentration in the sample was used to adjust the amount taken for the PCR reaction. Samples with up to $10 \mathrm{ng} / \mu \mathrm{l}$ were used as is; for $11-100 \mathrm{ng} / \mu \mathrm{l}$ the sample was diluted 1 volume sample plus 9 volumes PCR GRADE WATER (HyLabs, Cat number BP556/100S(, before taken to for PCR; for over $101 \mathrm{ng} / \mu \mathrm{l}$ the sample was diluted 1 volume sample plus 99 volume PCR GRADE WATER before taken for PCR. These adjustments helped overcome cases of PCR inhibition, mainly in vaginal swabs that gave extremely high yields of nucleic acids.

\section{Nested PCR for chlamydia trachomatis}

The method is based on two consecutive PCR reactions and sequencing of the MLST regions. In the first PCR (nested-1) we used external primers and in the second PCR (nested-2) we used more internally located primers. For sequencing we used a third set of primer that is located even more internally and was not involved in the PCR amplification step (Table 1, see supplementary information, Figures S1, S2, S3, S4, S5 and $\mathrm{S6}$ for sequence \& graphical presentation). StepOnePlus Real-Time PCR (Applied Biosystems, Waltham, MA, USA) was used for PCR amplification during this study. The amplification of each target gene was performed separately.

\section{Nested-1 PCR}

In the first PCR reaction, we used sets of external primers (HyLabs Israel LTD, Table 1) for DNA amplification on the regions of hctB (CT046), CT058, CT144, CT172, $p b p B$ (CT682) and ompA. The amplification was performed in a volume of $20 \mu \mathrm{l}$ containing $8 \mu \mathrm{l}$ of extracted DNA, $0.02 \mathrm{U} / \mu \mathrm{l}$ KOD Hot-Start DNA polymerase (EMD Millipore Corp., 71,086-3), $1.5 \mathrm{mM} \mathrm{MgSO}_{4}$, $0.2 \mathrm{mM}$ each deoxynucleoside triphosphate (dNTPs) and $0.5 \mu \mathrm{M}$ of each specific outer primer. The fluorescent dye LightCycler ${ }^{\circ} 480$ ResoLight Dye (Roche, 0490964001) was used in the reaction at final dilution $\mathrm{X} 40$. Cycling conditions were $120 \mathrm{~s}$ polymerase activation step at $95^{\circ} \mathrm{C}$, followed by 45 cycles of denaturation at $95^{\circ} \mathrm{C}$ for $20 \mathrm{~s}$, annealing at $53^{\circ} \mathrm{C}$ for $15 \mathrm{~s}$ and extension at $70^{\circ} \mathrm{C}$ for $20 \mathrm{~s}$ for $500-1000 \mathrm{bp}$ target. Melt curve: $95^{\circ} \mathrm{C}$ for $15 \mathrm{~s}, 60^{\circ} \mathrm{C}$ for $60 \mathrm{~s}$ and up to $95^{\circ} \mathrm{C}$, measuring light emission every $0.3 \mathrm{~s}$. C. trachomatis ATCC strain DNA diluted from 1:500 to 1:2000 was used as a positive DNA control.

\section{Nested-2 PCR}

The consecutive reaction was performed using $1 \mu \mathrm{l}$ of nested-1 reaction in a total volume of $10 \mu$. The reaction contained internal primers as described in Table 1, 0.02 $\mathrm{U} / \mu \mathrm{l} \mathrm{KOD} \mathrm{Hot-Start} \mathrm{DNA}$ polymerase, $1.5 \mathrm{mM} \mathrm{MgSO}_{4}$, $0.2 \mu \mathrm{M}$ each deoxynucleoside triphosphate and $0.5 \mu \mathrm{M}$ of each specific outer primer, LightCycler 480 ResoLight Dye at X40 final dilution. Cycling conditions were $120 \mathrm{~s}$ of polymerase activation step at $95^{\circ} \mathrm{C}$, followed by $40 \mathrm{cy}$ cles of denaturation at $95^{\circ} \mathrm{C}$ for $20 \mathrm{~s}$, annealing $51^{\circ} \mathrm{C}$ for $15 \mathrm{~s}$ and extension at $70^{\circ} \mathrm{C}$ for $20 \mathrm{~s}$. Melting curve analysis was performed as in nested-1.

\section{Cleaning PCR products}

At the end of nested-2 PCR reactions, the PCR reactions were cleaned by the Agencourt AMPure $\mathrm{XP}^{\circ}$ (Beckman Coulter, A63881) PCR purification system of paramagnetic bead technology. For each of the $10 \mu \mathrm{L}$ PCR reaction, $18 \mu \mathrm{L}$ of rigorously re-suspended beads were added. We separated the DNA from the beads using the Agencourt SPRIPlate Super Magnet Plate (Beckman Coulter A32782), followed by two washes with $100 \mu \mathrm{L}$ of $70 \%$ ethanol, and elution with $40 \mu \mathrm{l}$ of $1 \mathrm{xTE}$ solution (TrisEDTA buffer, 100x Concentrate, Sigma Aldrich, Cat \# T9285-100ML). The purified DNA was stored in $-20^{\circ} \mathrm{C}$ until use.

\section{Bom's MLST scheme}

The protocol is based on the primers described in Bom RJ et all., 2011 [28] with the use of the reaction condition exactly as described for our method.

\section{Sequencing analysis}

The amount of the purified DNA was measured using the PicoGreen or DeNovix assay as indicated and the DNA was diluted according to the HyLabs Israel LTD instructions prior sequencing. Each individual amplification product was cleaned and was sequenced from both sides, using the internal Seq_F and Seq_R primers (Table 1). Next, the sequences were examined with the Chromas ver. 2.6.5 (Technelysium DNA sequencing software) and DNA sequencing quality was inspected. Gene contigs were generated by uploading the forward and reverse sequences of each gene into Clone Manager 9.0 (Sci-Ed Software). Cases of disagreements between the forward and reverse sequences were resolved by using the best chromatogram. The assembled sequences were saved in a FASTA format. In order to get MLST Sequence Type (ST), we used the Uppsala, Sweden, C. trachomatis MLST database website (http://mlstdb.bmc.uu. se/current.html) [34] for sequences interrogation. For ompA genotyping we used Blastn at the NCBI database (https://blast.ncbi.nlm.nih.gov/Blast.cgi). 


\section{Developing of scoring system for prediction of PCR amplifications success}

We utilized the Real Time PCR Melt Curve (MC) and cycle threshold (CT) diagrams (obtained from the nested-2 PCR step) in order to predict amplification success. The melting curves were compared to those of the positive control. The amplifications were classified into three categories:

Good (G): $\mathrm{CT} \leq 20$, melting curve with a single peak with $\mathrm{Tm}$ within $1^{\circ} \mathrm{C}$ from the $\mathrm{Tm}$ of the positive control,

Bad (B): CT $>20$ and a single peak more than $3{ }^{\circ} \mathrm{C}$ from the Tm of the positive control or multiple peaks, Moderate $(\mathrm{M}): \mathrm{CT} \leq 20$ and a single dominant peak $1{ }^{\circ} \mathrm{C}-3^{\circ} \mathrm{C}$ from the $\mathrm{Tm}$ of the positive control and possibly a secondary non-specific peak or, CT $>20$, melting curve with a single peak with Tm within $1{ }^{\circ} \mathrm{C}$ from the Tm of the positive control (Fig. 2). For example, if the $\mathrm{MC}$ is identical to the positive control and the $\mathrm{CT} \leq 20$ in nested 2 step, the score will be Good (G), but if the $\mathrm{MC}$ represents multiple peaks, or a major peak with a Tm that is significantly different from the positive control (more than $3{ }^{\circ} \mathrm{C}$ ), the score will be Bad (B). For more examples, see Fig. 2B. The correlation between the amplification score $(\mathrm{G} / \mathrm{M} / \mathrm{B})$ to sequencing successes was determined.

\section{Limits of detection (LOD)}

We used ATCC certificate of analysis data of the following strains ATCC VR-885 (D), ATCC VR-878 (G) and ATCC VR-901BD (LGV1) in order to calculate the DNA copy number, using NEBioCalculator (http:// nebiocalculator.neb.com/\#!/dsdnaamt) according to the following formulas:

$$
\begin{aligned}
& \text { moles dsDNA }(\mathrm{mol}) \\
& \begin{aligned}
&=\text { mass of dsDNA }(\mathrm{g}) /((\text { length of dsDNA }(\mathrm{bp}) \times 617.96 \mathrm{~g} / \mathrm{mol})+36.04 \mathrm{~g} / \mathrm{mol}) \\
& \text { moles of dsDNA ends }=\text { moles dsDNA }(\mathrm{mol}) \times 2 \\
& \text { DNA copy number }= \text { moles of dsDNA } \\
& \times 6.022 \mathrm{e} 23 \text { molecules } / \mathrm{mol}
\end{aligned}
\end{aligned}
$$

Droplet Digital PCR (ddPCR) data of C. trachomatis DNA copy number was available from ATCC for strain ATCC VR-901BD. The actual DNA copy number (by ddPCR) was used for estimation of the actual copy number in strains ATCC VR-885, ATCC VR-878. The stock DNA was serially diluted in PCR GRADE WATER according to Table 4 and $8 \mu \mathrm{l}$ of each dilution was used for performing MLST as indicated. The amplification quality was scored according to our Good/Moderate/Bad scheme and only Good score was considered as successful amplification. In a similar experiment 912, 91.2 DNA copy number of ATCC VR-885, VR-901BD were spiked into $400 \mu \mathrm{l}$ of negative urine (as determined by GeneXpert). The DNA was purified using MagNa pure compact and the MLST was performed as indicated with a successful amplification (not presented).

\section{Comparative analysis}

Thirty-two urine samples that were found positive for C.trachomatis by GeneXpert test were chosen for the comparative study. For details about each sample see Table S8 in the supplementary section. These samples are part of the 81 urine sample that were used in this study (Table S9 in the supplementary section). The samples represent various DNA qualities, DNA concentration, as well as various genovars and STs. Each sample was analyzed once with the method presented in this study, or by Bom's MLST primers scheme [28].

\section{Supplementary Information}

The online version contains supplementary material available at https://doi. org/10.1186/s12866-020-02077-y.

\section{Additional file 1.}

\section{Abbreviation}

MLST: Multilocus Sequence Typing

\section{Acknowledgements \\ We are grateful to Dr. Paul Jeffrey Freidlin for proofreading the article and the smart suggestions.}

\section{Authors' contributions}

IN conceived the idea of study and model development. Experiments were performed by SP, LP, MR. Bioinformatics analysis were performed by MR, IN. IN, GZV, MR, SP, YS, ER wrote the manuscript. Revising of the manuscript was made by IN, SP, GZV, MR, ZD, ER. All authors read and approved the final manuscript.

\section{Funding}

This study was supported by the Israeli Ministry of Health.

\section{Availability of data and materials}

The datasets generated during the current study are available in the NCBI GenBank repository, under accession numbers, ompA: MW258775 MW258830; CT046 (hctB): MW309910 - MW309962; CT058: MW309963 MW310018; CT144: MW310075 - MW310130; CT172: MW310019 - MW310074; CT682 (pbpB): MW310131 - MW310186.

\section{Ethics approval and consent to participate}

This study was approved by the ethical committee at Edith Wolfson Medical Center, number 0075-19-WOMC. The committee follows the Helsinki declaration.

\section{Consent for publication}

Not applicable.

Competing interests

The authors declare that they have no competing interests. 
Received: 22 April 2020 Accepted: 20 December 2020

Published online: 18 January 2021

\section{References}

1. Bachmann NL, Polkinghorne A, Timms P. Chlamydia genomics: providing novel insights into chlamydial biology. Trends Microbiol. 2014;22(8):464-72.

2. Adachi K, Nielsen-Saines K, Klausner JD. Chlamydia trachomatis Infection in Pregnancy: The Global Challenge of Preventing Adverse Pregnancy and Infant Outcomes in Sub-Saharan Africa and Asia. Biomed Res Int. 2016;2016: 9315757.

3. Paavonen J, Eggert-Kruse W. Chlamydia trachomatis: impact on human reproduction. Hum Reprod Update. 1999;5(5):433-47.

4. Organization WH: Global health sector strategy on sexually transmitted infections, 2016-2021. 2016.

5. Malhotra M, Sood S, Mukherjee A, Muralidhar S, Bala M. Genital Chlamydia trachomatis: an update. Indian J Med Res. 2013;138(3):303-16.

6. Chesson HW, Pinkerton SD. Sexually transmitted diseases and the increased risk for HIV transmission: implications for cost-effectiveness analyses of sexually transmitted disease prevention interventions. J Acquir Immune Defic Syndr. 2000;24(1):48-56.

7. Buckner LR, Amedee AM, Albritton HL, Kozlowski PA, Lacour N, McGowin CL, Schust DJ, Quayle AJ. Chlamydia trachomatis Infection of Endocervical Epithelial Cells Enhances Early HIV Transmission Events. PLoS One. 2016; 11(1):e0146663.

8. Bom RJ, van der Helm JJ, van der Loeff MF S, van Rooijen MS, Heijman T, Matser A, de Vries HJ, Bruisten SM. Distinct transmission networks of Chlamydia trachomatis in men who have sex with men and heterosexual adults in Amsterdam, The Netherlands. PLoS One. 2013;8(1):e53869.

9. Harris SR, Clarke IN, Seth-Smith HM, Solomon AW, Cutcliffe LT, Marsh P, Skilton RJ, Holland MJ, Mabey D, Peeling RW, et al. Whole-genome analysis of diverse Chlamydia trachomatis strains identifies phylogenetic relationships masked by current clinical typing. Nat Genet. 2012;44(4):413-9 S411.

10. Pawlikowska-Warych M, Sliwa-Dominiak J, Deptula W. Chlamydial plasmids and bacteriophages. Acta Biochim Pol. 2015;62(1):1-6.

11. Seth-Smith HM, Harris SR, Skilton RJ, Radebe FM, Golparian D, Shipitsyna E, Duy PT, Scott P, Cutcliffe LT, O'Neill C, et al. Whole-genome sequences of Chlamydia trachomatis directly from clinical samples without culture. Genome Res. 2013;23(5):855-66.

12. Pedersen LN, Herrmann B, Moller JK. Typing Chlamydia trachomatis: from egg yolk to nanotechnology. FEMS Immunol Med Microbiol. 2009:55(2): 120-30.

13. Pedersen LN, Podenphant L, Moller JK. Highly discriminative genotyping of Chlamydia trachomatis using omp1 and a set of variable number tandem repeats. Clin Microbiol Infect. 2008;14(7):644-52.

14. Wang SP, Kuo CC, Grayston JT. A simplified method for immunological typing of trachoma-inclusion conjunctivitis-lymphogranuloma venereum organisms. Infect Immun. 1973;7(3):356-60.

15. Bebear C, de Barbeyrac B. Genital Chlamydia trachomatis infections. Clin Microbiol Infect. 2009;15(1):4-10.

16. Levy I, Gefen-Halevi S, Nissan I, Keller N, Pilo S, Wieder-Finesod A, Litchevski V, Shasha D, Kedem E, Rahav G. Delayed diagnosis of colorectal sexually transmitted diseases due to their resemblance to inflammatory bowel diseases. Int J Infect Dis. 2018;75:34-8.

17. Wolkerstorfer A, de Vries HJ, Spaargaren J, Fennema JS, van Leent EJ. Inguinal lymphogranuloma venereum in a man having sex with men: perhaps an example of the missing link to explain the transmission of the recently identified anorectal epidemic. Ned Tijdschr Geneeskd. 2004;148(51): 2544-6.

18. Witkin SS, Minis E, Athanasiou A, Leizer J, Linhares IM. Chlamydia trachomatis: the Persistent Pathogen. Clin Vaccine Immunol. 2017;24(10): e00203-17.

19. Geisler WM. Duration of untreated, uncomplicated Chlamydia trachomatis genital infection and factors associated with chlamydia resolution: a review of human studies. J Infect Dis. 2010;201(Suppl 2):S104-13.

20. Rawre J, Juyal D, Dhawan B. Molecular typing of Chlamydia trachomatis: An overview. Indian J Med Microbiol. 2017;35(1):17-26.

21. Brunelle BW, Sensabaugh GF. The ompA gene in Chlamydia trachomatis differs in phylogeny and rate of evolution from other regions of the genome. Infect Immun. 2006;74(1):578-85.
22. Dean D, Millman K. Molecular and mutation trends analyses of omp1 alleles for serovar E of Chlamydia trachomatis. Implications for the immunopathogenesis of disease. J Clin Invest. 1997;99(3):475-83.

23. Kapil R, Press CG, Hwang ML, Brown L, Geisler WM. Investigating the epidemiology of repeat Chlamydia trachomatis detection after treatment by using C. trachomatis OmpA genotyping. J Clin Microbiol. 2015;53(2):546-9.

24. Herrmann B, Isaksson J, Ryberg M, Tangrot J, Saleh I, Versteeg B, Gravningen K, Bruisten S. Global Multilocus Sequence Type Analysis of Chlamydia trachomatis Strains from 16 Countries. J Clin Microbiol. 2015;53(7):2172-9.

25. Abdelsamed H, Peters J, Byrne Gl. Genetic variation in Chlamydia trachomatis and their hosts: impact on disease severity and tissue tropism. Future Microbiol. 2013;8(9):1129-46.

26. Joseph SJ, Read TD. Genome-wide recombination in Chlamydia trachomatis Nat Genet. 2012;44(4):364-6.

27. Patino LH, Camargo M, Munoz M, Rios-Chaparro DI, Patarroyo MA, Ramirez JD. Unveiling the Multilocus Sequence Typing (MLST) Schemes and Core Genome Phylogenies for Genotyping Chlamydia trachomatis. Front Microbiol. 2018;9:1854

28. Bom RJ, Christerson L, van der Loeff MF S, Coutinho RA, Herrmann B, Bruisten SM. Evaluation of high-resolution typing methods for Chlamydia trachomatis in samples from heterosexual couples. J Clin Microbiol. 2011; 49(8):2844-53.

29. Borges V, Ferreira R, Nunes A, Sousa-Uva M, Abreu M, Borrego MJ, Gomes JP. Effect of long-term laboratory propagation on Chlamydia trachomatis genome dynamics. Infect Genet Evol. 2013;17:23-32.

30. de Vries HJ, van der Loeff MF S, Bruisten SM. High-resolution typing of Chlamydia trachomatis: epidemiological and clinical uses. Curr Opin Infect Dis. 2015;28(1):61-71.

31. Putman TE, Suchland RJ, Ivanovitch JD, Rockey DD. Culture-independent sequence analysis of Chlamydia trachomatis in urogenital specimens identifies regions of recombination and in-patient sequence mutations. Microbiology. 2013;159(Pt 10):2109-17.

32. Pannekoek Y, Morelli G, Kusecek B, Morre SA, Ossewaarde JM, Langerak AA, van der Ende A. Multi locus sequence typing of Chlamydiales: clonal groupings within the obligate intracellular bacteria Chlamydia trachomatis. BMC Microbiol. 2008;8:42.

33. Dean D, Bruno WJ, Wan R, Gomes JP, Devignot S, Mehari T, de Vries HJ, Morre SA, Myers G, Read TD, et al. Predicting phenotype and emerging strains among Chlamydia trachomatis infections. Emerg Infect Dis. 2009; 15(9):1385-94.

34. Jolley KA, Bray JE, Maiden MCJ. Open-access bacterial population genomics: BIGSdb software, the PubMLST.org website and their applications. Wellcome Open Res. 2018;3:124.

35. Ikryannikova LN, Shkarupeta MM, Shitikov EA, Il'ina EN, Govorun VM. Comparative evaluation of new typing schemes for urogenital Chlamydia trachomatis isolates. FEMS Immunol Med Microbiol. 2010;59(2):188-96.

36. Klint M, Fuxelius HH, Goldkuhl RR, Skarin H, Rutemark C, Andersson SG, Persson K, Herrmann B. High-resolution genotyping of Chlamydia trachomatis strains by multilocus sequence analysis. J Clin Microbiol. 2007; 45(5):1410-4.

37. Giffard PM, Andersson P, Wilson J, Buckley C, Lilliebridge R, Harris TM, Kleinecke M, O'Grady KF, Huston WM, Lambert SB, et al. CtGEM typing: Discrimination of Chlamydia trachomatis ocular and urogenital strains and major evolutionary lineages by high resolution melting analysis of two amplified DNA fragments. PLoS One. 2018;13(4):e0195454.

38. Gharsallah H, Frikha-Gargouri O, Bom RJ, Hammami A, Bruisten SM. Comparison of reverse hybridization and ompA sequencing methods applied on Chlamydia trachomatis strains from Tunisia. Microbiologyopen. 2018;7(2):e00549.

39. Zhou Y, Cai YM, Li SL, Cao NX, Zhu XF, Wang F, Han Y, Yin YP, Chen XS. Anatomical site prevalence and genotypes of Chlamydia trachomatis infections among men who have sex with men: a multi-site study in China. BMC Infect Dis. 2019;19(1):1041.

40. Rawre J, Dhawan B, Malhotra N, Sreenivas V, Broor S, Chaudhry R. Prevalence and distribution of Chlamydia trachomatis genovars in Indian infertile patients: a pilot study. APMIS. 2016;124(12):1109-15.

\section{Publisher's Note}

Springer Nature remains neutral with regard to jurisdictional claims in published maps and institutional affiliations. 